\title{
Novel QTL For Lodging Resistance, PRL4, Improves Physical Properties With High Non-Structural Carbohydrate Accumulation of Basal Culms in Rice (Oryza Sativa L.)
}

Takayuki Kashiwagi ( $\nabla$ kashiwagi@cc.utsunomiya-u.ac.jp )

Utsunomiya University https://orcid.org/0000-0002-0096-8113

\section{Research Article}

Keywords: Rice, Lodging resistance, Pushing resistance, Culm physical property, Non-structural carbohydrate

Posted Date: August 17th, 2021

DOI: https://doi.org/10.21203/rs.3.rs-792664/v1

License: (c) (1) This work is licensed under a Creative Commons Attribution 4.0 International License. Read Full License 


\section{Abstract}

Resistance to lodging, an important problem in rice production, has three types: low plant height, strong culm, and high strength of the lower part of the plant. The determinants of strength of the lower part remains unclear, compared with plant height and culm strength. This study identified a new genetic factor involved in the strength of the lower part, as assessed by pushing resistance, using chromosomal segment substitution lines (CSSLs) to clarify the determinants of strength of the lower part by functional analysis of the CSSL and the near isogenic line (NIL) harboring the identified quantitative trait locus (QTL). QTL analysis identified the QTL for increasing pushing resistance on chromosome 4, PRL4, which was not related to days to heading. The CSSL with PRL4 showed increased pushing resistance and physical strength of the basal culm, but decreased filled grain ratio and grain weight. The NIL with PRL4, developed by backcrossing this CSSL, improved pushing resistance and the strain of culm until breaking under compression, and did not decrease yield traits. These lines with PRL 4 increased the accumulation of non-structural carbohydrate (NSC) in the basal culm at the fully ripe stage. Thus, the genetic control of NSC accumulation in culms by PRL4 may improve the strength of the lower part by enhancing culm toughness with strength and ductility.

\section{Introduction}

Lodging is an important problem in rice production that causes reductions in yield, grain quality, and mechanical harvesting efficiency (Kono, 1995). Resistance to lodging has three types: low plant height, strong culm, and high strength of the lower part of the plant. Short stature is the first-generation target for lodging resistance; e.g., the "IR" cultivars developed by the International Rice Research Institute (Peng et al. 1999). Strong culm is the second target for lodging resistance. The new plant type lines with high lodging resistance have thick and sturdy culms (Peng et al. 1999). "Leaf Star" and "Monster rice 1" have long culms, while these lines show greater lodging resistance via high section modulus and bending moment at breaking in the culms (Ookawa et al. 2010b; Nomura et al. 2019). The strong culm phenotype is mainly contributed by culm morphological traits; thus, culm diameter, culm wall thickness, and thickness of cortical fiber tissue are key targets for the improvement of lodging resistance.

Several genetic factors affecting the relationship between physical properties and morphological traits in culms have been reported in rice. The QTL for stem diameter on chromosome 8, sdm8, increases culm wall thickness and culm stiffness (Kashiwagi et al. 2008). The strong culm QTL SCM2, which is identical to ABERRANT PANICLE ORGANIZATION1 (APO1), increases section modulus by thickening the culm diameter and culm wall (Ookawa et al. 2010a; Yano et al. 2015). SCM3, identified on chromosome 3 , causes a thick culm diameter and high section modulus and has a greater bending moment at breaking than SCM2 (Yano et al. 2015). Furthermore, the pyramiding line with SCM2 and SCM3 shows a greater bending moment at breaking and section modulus compared with the NIL for each QTL. In addition, the candidate gene OSPRR37 contributes to increases in breaking strength, culm wall thickness, and culm diameter (Guo et al. 2021), and the gw2 mutant, which has a large grain, increases culm diameter and improves lodging resistance (Yamauchi et al. 2020). On the other hand, chemical components of culms, 
especially carbohydrate accumulation, affect their physical properties. Structural carbohydrates cellulose and lignin are important components for culm strength. The $b s 3$ and $f c 1$ mutants decrease cellulose and lignin content, respectively, and cause thin cell wall thickness and a weak culm (Hirano et al. 2010; Li et al. 2009). BSUC11 increases the holocellulose content of the upper culms at the fully ripe stage and increases breaking strength without altering culm morphological traits (Kashiwagi et al. 2016). In other carbohydrates, Ishimaru et al. (2008) reported that Irt5 enhances the Young's modulus of the upper culms, an indicator of rigidity, by increasing starch content. Culm physical strength can be improved by the genetic control of morphological traits or chemical components of the culm; therefore, a second generation for lodging resistance, with the strong culm phenotype, should be developed using this genetic factor in the future.

There are still many unknowns about the third target for lodging resistance, strength of the lower part of the plant, as compared with plant height and culm strength. The strength of the lower part is often evaluated using the pushing resistance method. Pushing resistance correlates with the diameter and weight of culms as well as root morphology (Kashiwagi et al. 2008; Terashima et al. 1994). Several genetic factors for pushing resistance have been reported in rice. QTLs for pushing resistance, as evaluated by a single-stem anti-thrust value, have been detected on chromosome 1, 2, 3, 6, and 8 (Sowadan et al. 2018). Merugumala et al. (2019) detected QTLs for pushing resistance as bending stress on chromosome 2. From the backcross inbred lines of japonica "Nipponbare" and indica "Kasalath", the QTL for pushing resistance of the lower part, prl5, which was measured by removing the upper part of the plant, was identified. Studies have shown that prl5 increases non-structural carbohydrate (NSC) accumulation in the culms at the fully ripe stage by delaying leaf senescence (Kashiwagi and Ishimaru, 2004; Kashiwagi et al. 2006). In addition, Fan et al. (2018) reported that OsEXTL overexpression thickens the secondary cell wall, increases pushing resistance, and improves lodging resistance. Pushing resistance is determined by the morphological traits and chemical components of the culms or root morphology. The relationships between physical strength and morphological traits or chemical components in culms are similar to the determinants of pushing resistance; however, high culm strength does not always correspond to high pushing resistance. For example, sdm8 and BSUC11 increase culm stiffness and breaking strength, respectively, but two loci do not contribute to improvement of pushing resistance (Kashiwagi et al. 2008; Kashiwagi, 2014). These results suggest that there are different determinants for pushing resistance and culm physical properties. Therefore, more detailed functional analysis on the pushing resistance and physical properties of the basal culm are needed to improve the strength of the lower part.

Functional analysis of genetic factors is a useful tool for clarifying the mechanism of target determination. Identifying new genetic factors in the strength of the lower part and clarifying their functions will help to identify the determinants of this strength in rice lodging resistance. This study identifies a new genetic factor involved in pushing resistance using the chromosomal segment substitution lines (CSSLs) and clarifies the determinants of strength of the lower part from its functional analysis. 


\section{Materials And Methods}

Plant materials

CSSLs with chromosomal segments of "Nona Bokra" with the genetic background of "Koshihikari", developed by Takai et al. (2007), were used for QTL analysis from 2016 to 2018 . Seeds of 44 CSSLs and "Koshihikari" were sown in a greenhouse in late April 2016 and 2017, and four CSSLs with the chromosomal segment on chromosome 4 (line nos. 511-514) were sown in 2018. In late May, 10 plants were transplanted, with a single plant per hill spaced at $30 \times 15 \mathrm{~cm}$, into a paddy field in Utsunomiya, Japan (latitude $36^{\circ} \mathrm{N}$, longitude $139^{\circ} \mathrm{E}$ ), and grown under natural conditions. For QTL characterization, CSSL512 (CSSL-PRL4) and "Koshihikari" were grown in each plot of 10 rows with 12 hills per row in 2017 and 2018. These seeds were sown in late April and transplanted to a single plant per hill, spaced at $30 \times$ $15 \mathrm{~cm}$, in late May. In 2019 and 2020, the NIL with PRL4 (NIL-PRL4) and "Koshihikari" was grown under the same cultivation conditions as in 2017 and 2018. Additionally, NIL-LPW4 was grown in each plot of two rows, with 12 hills per row, in 2019 and 2020. N, $\mathrm{P}_{2} \mathrm{O}_{5}$, and $\mathrm{K}_{2} \mathrm{O}$ fertilizers were applied as basal dressing at $6 \mathrm{~g} \mathrm{~m}^{-2}$ before transplanting, respectively, in 2016-2020.

QTL analysis

QTLs for pushing resistance of the lower part per plant or tiller, tiller number, and days to heading were determined by comparing the phenotypes and the genotypes of 140 simple sequence repeats and five expressed sequence tag markers (Takai et al. 2007). QTL detection was based on a $t$-test, with the threshold at a probability level of 0.05 , of the difference between each CSSL and "Koshihikari", according to Madoka et al. (2008). The additive effect of a QTL from the "Nona Bokra" allele was expressed as positive (plus) or negative (minus) relative percentage compared with the "Koshihikari" phenotype.

Selection of CSSL and NIL with PRL4

From 44 CSSLs, only CSSL512, carrying 10.3 Mb of the "Nona Bokra" homozygous allele, was selected to characterize the QTL effect for the PRL4 position on chromosome 4. After the backcross of CSSL512 with "Koshihikari", the genotypes of $288 \mathrm{BC}_{1} \mathrm{~F}_{2}$ plants were analyzed using eight SSR markers (International Rice Genome Sequencing Project, 2005). NIL-PRL 4 was selected from $288 \mathrm{BC}_{1} \mathrm{~F}_{3}$ plants by genotyping using 13 SSR markers. NIL-PRL4 had $6.6 \mathrm{Mb}$ of the "Nona Bokra" homozygous allele, localized nearest PRL4 marker RM5749 (Fig. 3a). In addition, because the locus for low panicle weight, which was named LPW4 in this study, was identified at the nearest marker RM3308 by Ujiie et al. (2012), NIL-LPW4, carrying 3.2 Mb of "Nona Bokra" homozygous allele between RM7113 and RM3308, was selected from the $\mathrm{BC}_{1} \mathrm{~F}_{3}$ plants (Supplementary Fig. 1a). DNA extraction was conducted using TPS buffer according to Monna et al. (2002). PCR was carried out using KAPA Taq Extra (Kapa Biosystems, USA), and the PCR profile was $94^{\circ} \mathrm{C}$ for $2 \mathrm{~min}, 35 \mathrm{cycles}$ of $94^{\circ} \mathrm{C}$ for $30 \mathrm{sec}, 55^{\circ} \mathrm{C}$ for $15 \mathrm{~s}, 72^{\circ} \mathrm{C}$ for $30 \mathrm{~s}$, and a final extension at $72^{\circ} \mathrm{C}$ for $5 \mathrm{~min}$. The resultant PCR products were visualized using $4 \%$ agarose gel stained with Midori Green Advance (Nippon Genetics, Tokyo, Japan). 
At seven weeks after heading (7WAH), the pushing resistance of the lower part (PRL) was analyzed according to Kashiwagi and Ishimaru (2004). The upper part of each plant was removed at a height of 40 $\mathrm{cm}$, and a prostrate tester (Daiki Rika Kogyou Co., Tokyo, Japan) was placed vertically to the culm at a height of $20 \mathrm{~cm}$ above the soil surface. The pushing resistance was measured until the plants had inclined to an angle of $45^{\circ}$ vertically. The pushing resistance of the lower part per tiller (PRL/TN) was calculated after measurement of tiller number. Physical properties of a basal internode were analyzed using universal table-top testing instruments (EZ-SX, Shimadzu, Kyoto, Japan). An average-sized basal internode was compressed with a compression jig (1 mm radius $\times 80 \mathrm{~mm}$ width) at a constant velocity of $1 \mathrm{~mm} \mathrm{~min}{ }^{-1}$, and compression resistance, strain at the maximum compression resistance, and culm stiffness were measured. The angle of each plant to the ground was measured using a protractor under field conditions. All lodging resistance traits were determined using six plants in each line.

Morphological traits, SPAD value of leaves, and yield components

Plant length and tiller number were measured before the harvest season, and the lengths and diameters of six average basal internodes per plant were measured at 7WAH. In 2018, basal internodes of "Koshihikari" and CSSL-PRL4 were measured to determine the thicknesses of the culm wall and cortical fiber tissue. Sampled basal culms were dried at $80^{\circ} \mathrm{C}$ for $3 \mathrm{~d}$, and the dried weight per unit length was calculated. SPAD values of the top three leaves were measured using a chlorophyll meter (SPAD-502, KONICA MINOLTA Inc., Tokyo, Japan) immediately after heading and at 7WAH. Measurements of morphological traits and SPAD values were conducted in six replicates. The panicles of six plants from each line were randomly sampled at $7 \mathrm{WAH}$, and the yield components were measured at the individual plant level.

\section{Carbohydrate content of basal culms}

Dried basal culms were ground to a powder using a Wonder Blender (Osaka Chemical Co., Osaka, Japan). Holocellulose content was measured in accordance with the sodium chlorite method of Wise et al. (1946). A powdered sample of $0.3 \mathrm{~g}$ was degreased in ethanol-benzene $(1: 2 \mathrm{v} / \mathrm{v})$ with a Soxhlet extraction for $6 \mathrm{~h}$ and then air-dried. After drying at $80^{\circ} \mathrm{C}$ for $1 \mathrm{~h}$, the sample was added to hot distilled water at $80^{\circ} \mathrm{C}$ and delignified by the addition of sodium chlorite and glacial acetic acid at $80^{\circ} \mathrm{C}$ for $1 \mathrm{~h}$. This process was repeated four times. The sample solution was filtrated through a glass filter, and the residue was washed in cool distilled water and acetone. After drying at $105^{\circ} \mathrm{C}$ for $1 \mathrm{~h}$, the residue was cooled to room temperature in a desiccator and the holocellulose content was weighed. Lignin content was measured in accordance with Suzuki et al. (2009). A powdered sample of approximately $15 \mathrm{mg}$ was transferred to a microcentrifuge tube with a screw cap and dried at $60^{\circ} \mathrm{C}$ for $1 \mathrm{~h}$. The sample was extracted with distilled water and centrifuged for $10 \mathrm{~min}$ at $16,200 \times \mathrm{g}$. The pellet was extracted in methanol at $60^{\circ} \mathrm{C}$ for $20 \mathrm{~min}$ and then centrifuged for $10 \mathrm{~min}$ at $16,200 \times \mathrm{g}$. This process was then repeated. After drying with a rotary evaporator (CC-105, TOMY, Tokyo, Japan), $3 \mathrm{M} \mathrm{HCl}$ and thioglycolic 
acid were added to the pellet. The sample was heated at $80^{\circ} \mathrm{C}$ for $3 \mathrm{~h}$ and centrifuged for $10 \mathrm{~min}$ at $16,200 \times \mathrm{g}$. The pellet was vortexed in distilled water and resuspended in $1 \mathrm{M} \mathrm{NaOH}$ for $16 \mathrm{~h}$ with a culture rotator (RT-50, TAITEC, Saitama, Japan). After centrifugation, the supernatant was acidified with concentrated $\mathrm{HCl}$ and chilled at $4^{\circ} \mathrm{C}$ for $4 \mathrm{~h}$. The sample was then centrifuged and the pellet was dissolved and diluted with $1 \mathrm{M} \mathrm{NaOH}$. The absorbance at $280 \mathrm{~nm}$ of diluted TGAL solution was recorded using a V-730BIO spectrophotometer (JASCO, Tokyo, Japan). NSC content was measured in accordance with Ishimaru et al. (2001). A powdered sample of approximately $20 \mathrm{mg}$ was extracted twice with $80 \%$ $(\mathrm{v} / \mathrm{v})$ ethanol at room temperature, once with $80 \%$ ethanol at $80^{\circ} \mathrm{C}$, and then centrifuged at $2,400 \times \mathrm{g}$ for 5 min. The supernatant was collected and dried in a vacuum, and then the sample was dissolved in distilled water, centrifuged at $14,000 \times g$ for $5 \mathrm{~min}$, and used for the determination of sucrose and hexoses by an enzymatic method (Bergmeyer and Bernt 1974). To determine starch content, the pellet was boiled in distilled water for $1 \mathrm{~h}$ and then digested with amyloglucosidase for $20 \mathrm{~min}$ at $55^{\circ} \mathrm{C}$. The resultant hexoses were determined by the enzymatic method. Six plants from each line were used to determine carbohydrate content.

\section{Statistical analysis}

Comparisons between "Koshihikari" and the CSSLs or NIL-PRL4 were carried out using a $t$-test at a probability of $P<0.05$ only when the F-test showed significance at 0.05 probability levels with Microsoft Excel 2018 for Mac.

\section{Results}

Pushing resistances in CSSLs and correlation with days to heading

Pushing resistance of the lower part (PRL) of "Koshihikari" was $5.80 \pm 0.67 \mathrm{kPa} \mathrm{cm}^{-2}$, and pushing resistance of the lower part per tiller (PRL/TN) was $0.51 \pm 0.02 \mathrm{kPa} \mathrm{cm}^{-2}$ in 2016 (Fig. 1a, d). In the CSSLs, the maximum PRL and PRL/TN were 2.82 and 3.45 times those of "Koshihikari", and the minimum pushing resistances were 0.68 and 0.75 times, respectively. In 2017, the PRL and PRL/TN of "Koshihikari" were $4.41 \pm 0.37$ and $0.38 \pm 0.04 \mathrm{kPa} \mathrm{cm}^{-2}$, respectively (Fig. 1b, e). The maximum PRL and $\mathrm{PRL} / \mathrm{TN}$ were 3.45 and 4.18 times those of "Koshihikari", and the minimum pushing resistances were 0.66 and 0.71 times, respectively. In the CSSLs, CSSL508 showed the latest heading dates, which were 30 and $50 \mathrm{~d}$ later than those of "Koshihikari" in 2016 and 2017, respectively (data not shown). Heading dates of CSSL519 to 522, containing the chromosomal segments from "Nona Bokra" on chromosome 6, were later than those of "Koshihikari" (10 to 50 d delay, data not shown). For PRL and days to heading, there were significant correlations in 2016 and 2017 ( $r=0.79$ and 0.89, respectively; Fig. 1c, f). PRL/TN similarly showed significant correlations with days to heading ( $r=0.77$ and 0.85 in 2016 and 2017, respectively).

QTL analysis 
Eight QTLs for PRL were detected in 2016 or 2017, and two QTLs on chromosome 4 and 6 increased PRL in both years (Supplementary Table 1). A QTL on chromosome 4, PRL4, located the nearest marker RM5749, and the analysis in 2018 confirmed this locus. Two QTLs for PRL/TN on chromosome 3 and 6 showed positive effects in both years, along with the QTL on chromosome 3, PRLT3, located at the nearest marker RM5626. The locus for both PRL and PRL/TN was detected between RM4128 and RM5963 on chromosome 6. On chromosome 1, a QTL with a negative effect on tiller number was detected in both years. Six QTLs for days to heading were detected on chromosomes 3, 5, 7, 8, and 12, and these were shown to delay heading date. On chromosome 3, PRLT3 overlapped with the QTL for days to heading, $D H 3$ (Fig. 2). Four CSSLs with a chromosomal segment on chromosome 6 showed a delay in heading date, but the location of the QTL for days to heading could not be determined by the method of QTL analysis used in this study.

Selection of PRL4 and functional analysis using CSSL-PRL4

This study found three loci that improved pushing resistance in two separate years (Fig. 2). PRLT3 overlapped with $D H 3$, and CSSLs with PRL6 and PRLT6 showed a delay in heading date (data not shown). The delay in heading date significantly affected pushing resistance (Fig. 1c, f), while only PRL 4 did not contribute to heading date. For this reason, PRL 4 was targeted as a useful QTL for lodging resistance.

In basal culms, CSSL-PRL4 had the same length and diameter as "Koshihikari", but had 1.37 times greater dry weight (Supplementary Table 2). Additionally, CSSL-PRL4 had similar thicknesses of culm wall and cortical fiber tissue as the basal culms of "Koshihikari" (Supplementary Table 3). Compression resistances of CSSL-PRL4 were 1.26 and 1.18 times higher than those of "Koshihikari" in 2017 and 2018, respectively. In 2017, the culm stiffness of CSSL-PRL4 was 1.22 times higher than that of "Koshihikari", while its strain at the maximum compression resistance of CSSL-PRL4 was 1.38 times higher in 2018. CSSL-PRL4 showed the same content of structural carbohydrates in basal culms than "Koshihikari" (Supplementary Table 2). CSSL-PRL4 showed significantly higher NSC content in the basal culms than "Koshihikari". In the three types of non-structural carbohydrates, the starch content of CSSL-PRL 4 was 1.86 and 1.96 times higher than that of "Koshihikari" in 2017 and 2018, respectively. CSSL-PRL4 showed the same plant length and tiller number as "Koshihikari", and plant angles on the field in CSSL-PRL4 were greater than those of "Koshihikari" (Supplementary Table 4). Yield per plant of CSSL-PRL4 was 0.58 times that of "Koshihikari" in both years. In particular, CSSL-PRL4 showed a significant decrease in filled grain ratio. At 7WAH, the upper leaves of CSSL-PRL4 had a significantly higher SPAD value than those of "Koshihikari" in 2017, but both lines showed similar SPAD values in 2018.

Functional analysis in NIL-PRL4

PRLs in NIL-PRL4 were 1.60 and 1.57 times higher than those in "Koshihikari" (Fig. 3b). Similarly, NILPRL4 showed significantly higher PRL/TN than "Koshihikari". In 2019, NIL-PRL4 had greater diameter and dry weight of the basal culm, but both lines were similar in 2020 (Table 1). With regard to physical properties of the basal culm, NIL-PRL4 showed higher compression resistance than "Koshihikari" in 2019, 
but decreased culm stiffness in 2020. Strains at the maximum compression resistance of NIL-PRL4 were 1.18 and 1.22 times greater than those of "Koshihikari" in 2019 and 2020, respectively. The NSC content of basal culms in NIL-PRL4 was 1.27 and 1.24 times higher than that of "Koshihikari". NIL-PRL4 showed higher starch and hexose content than "Koshihikari" in 2019 and significantly increased sucrose content in 2020. In both years, NIL-PRL4 showed the same plant length and tiller number as "Koshihikari" and greater plant angles in the field (Table 2). The yield per plant of NIL-PRL4 was similar to that of "Koshihikari". Only the filled grain ratio of NIL-PRL 4 was 0.82 times of "Koshihikari" in 2020, while the other yield components of both lines were the same. Panicle weights of NIL-LPW4 were 0.80 and 0.85 times those of "Koshihikari" in 2019 and 2020, respectively (Supplementary Fig. 1b). Similarly, NIL-LPW4 showed a significantly lower ratio of filled grains compared to "Koshihikari" (0.71 and 0.76 times in 2019 and 2020, respectively). 
Table 1

Morphological traits, physical properties, and NSC contents in basal internodes of Koshihikari and NILPRL4

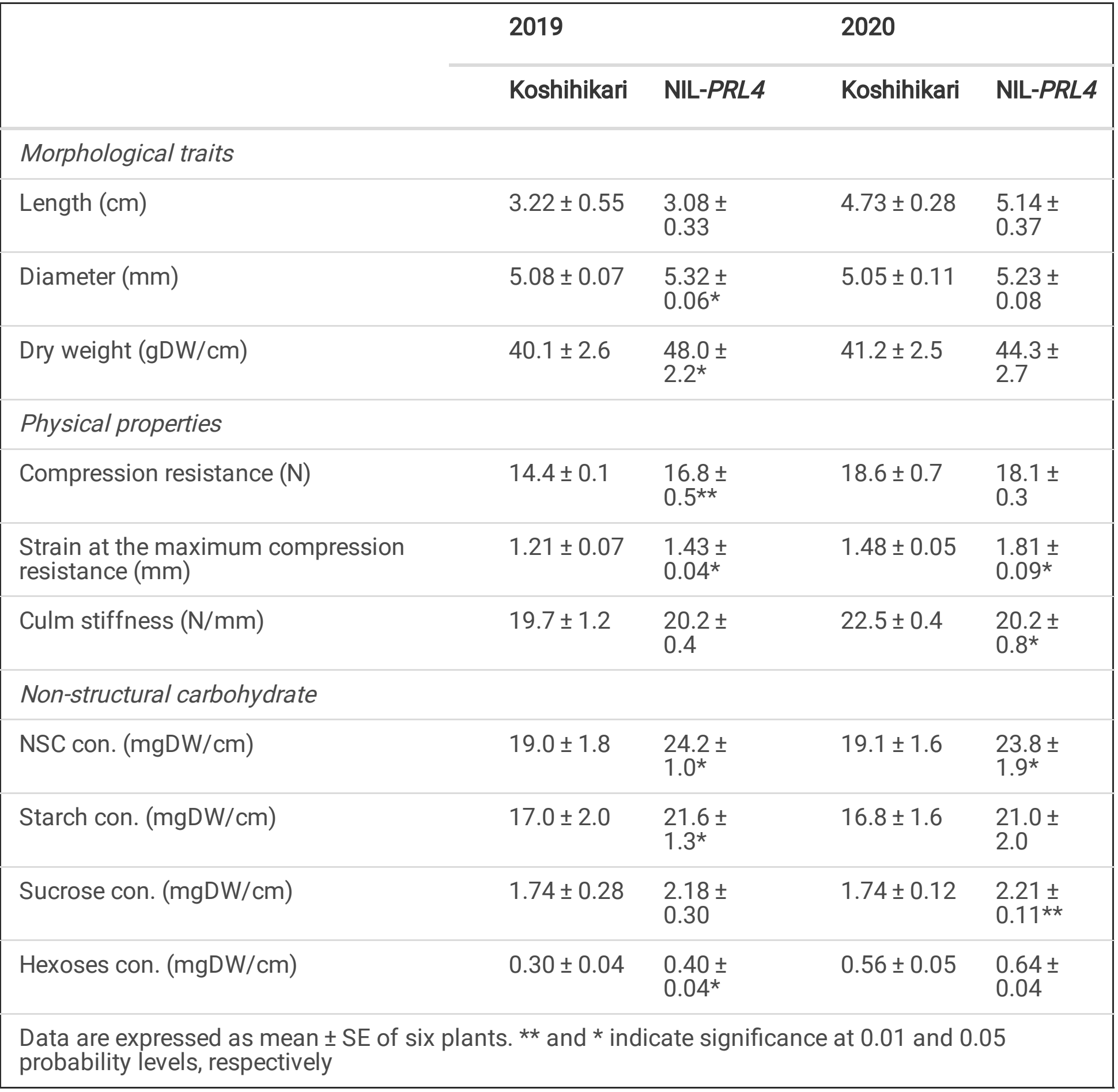


Table 2

Morphological traits, plant angle, yield components, and SPAD values of Koshihikari and NIL-PRL4

\begin{tabular}{|c|c|c|c|c|}
\hline \multirow[t]{2}{*}{ Traits } & \multicolumn{2}{|l|}{2019} & \multicolumn{2}{|l|}{2020} \\
\hline & Koshihikari & NIL-PRL4 & Koshihikari & NIL-PRL4 \\
\hline Plant length (cm) & $117.3 \pm 1.1$ & $114.7 \pm 1.1$ & $123.9 \pm 1.3$ & $125.9 \pm 0.9$ \\
\hline Tiller no. & $10.3 \pm 0.5$ & $10.2 \pm 0.6$ & $10.2 \pm 0.7$ & $9.7 \pm 0.6$ \\
\hline Plant angle $\left({ }^{\circ}\right)$ & $70.0 \pm 1.8$ & $75.8 \pm 1.5^{\star}$ & $62.2 \pm 1.4$ & $72.3 \pm 1.1^{\star \star \star}$ \\
\hline \multicolumn{5}{|l|}{ Yield component } \\
\hline Panicle no. & $10.3 \pm 0.6$ & $9.8 \pm 0.7$ & $9.3 \pm 0.3$ & $9.5 \pm 0.4$ \\
\hline Grain no. per panicle & $126.5 \pm 5.1$ & $142.3 \pm 5.7$ & $126.8 \pm 5.5$ & $137.6 \pm 6.2$ \\
\hline Filled grain ratio (\%) & $83.0 \pm 1.9$ & $85.5 \pm 2.0$ & $93.7 \pm 0.8$ & $76.8 \pm 3.8^{\star \star}$ \\
\hline 1000 grains wt. (g) & $20.8 \pm 0.2$ & $20.7 \pm 0.1$ & $21.0 \pm 0.2$ & $20.7 \pm 0.1$ \\
\hline Yield per plant (g) & $22.8 \pm 2.2$ & $24.8 \pm 2.1$ & $23.2 \pm 1.1$ & $20.7 \pm 1.5$ \\
\hline \multicolumn{5}{|l|}{$S P A D$ value at $7 W A H$} \\
\hline Flag leaf blade & $22.6 \pm 1.4$ & $21.2 \pm 1.0$ & $16.4 \pm 1.0$ & $21.2 \pm 1.3^{\star}$ \\
\hline Second leaf blade & $18.2 \pm 1.9$ & $17.4 \pm 0.5$ & $10.0 \pm 1.3$ & $14.9 \pm 1.5^{\star}$ \\
\hline Third leaf blade & $12.2 \pm 0.9$ & $12.7 \pm 0.4$ & $7.9 \pm 1.0$ & $10.6 \pm 1.0$ \\
\hline
\end{tabular}

\section{Discussion}

This study found three QTLs causing increased pushing resistance on chromosome 3, 4, and 6; of these, only PRL 4 did not relate to heading date (Fig. 2). Pushing resistance and heading date showed a high positive correlation (Fig. 1C, f); therefore, it is important to consider the effect of heading date in improving the strength of the lower part. Seven QTLs for pushing resistance on chromosomes 1, 2, 3, 6 and 8 , evaluated by a single-stem anti-thrust value, have been identified by genome-wide association mapping (Sowadan et al. 2018), and QTLs for pushing resistance as bending stress have been detected on chromosome 2 (Merugumala et al. 2019). In addition, from the backcrossed inbred lines of japonica "Nipponbare" and indica "Kasalath", five QTLs for pushing resistance have been detected on chromosomes 4, 5, 6, 11, and 12 (Kashiwagi and Ishimaru, 2004). In the present study, the QTL on chromosome 4, which decreased pushing resistance by the "Kasalath" allele, was located at nearest marker C946, and this location did not correspond with PRL4. These results indicate that PRL 4 is a novel 
QTL for pushing resistance, making it a new genetic target for improving lodging resistance without affecting heading date.

This study conducted functional analyses using the CSSL harboring PRL4 and the NIL developed by backcrossing this CSSL. CSSL-PRL4 showed greater pushing resistance with increasing compression resistance and dry weight of basal culms (Supplementary Table 2). The increase in basal culm weight was caused by high accumulation of NSC, mainly starch (Supplementary Table 2). Because there were no differences in morphological traits and structural carbohydrate content between CSSL-PRL 4 and "Koshihikari", PRL 4 may be responsible for the high starch content in the basal culms. Among yield traits, filled grain ratio and grain weight of CSSL-PRL4 were decreased (Supplementary Table 4). This negative effect might be caused by a reduction in NSC translocation from culm to grains. On chromosome 4, Ujiie et al. (2012) detected a QTL for decreasing panicle weight from same CSSLs used in this study. This locus, LPW4, was harbored in the chromosomal segment from "Nona Bokra" in CSSL-PRL4. NIL-PRL4, which was removed from the $L P W 4$ region of "Nona Bokra", did not show significant decreases in filled grain ratio or grain weight (Table 2). However, NIL-PRL4 increased the NSC content of the basal culms and improved pushing resistance (Table 1, Fig. 3). The QTL for pushing resistance identified from "Kasalath", pr/5, increases NSC re-accumulation of the culms without reducing yield after maturity; additionally, prl5 is responsible for a delay in leaf senescence (Kashiwagi and Ishimaru, 2004; Kashiwagi et al. 2006). In this study, the CSSL and the NIL harboring PRL 4 did not show delay of leaf senescence; therefore, PRL4 would have a different function in NSC accumulation of culms at the fully ripe stage. These results suggest that PRL 4 might partly suppress NSC translocation from culm to grains after heading, but would not affect yield.

The physical strength of the lower part in rice is determined by complex traits. PRL 4 significantly improved the physical strength of the lower part, as evaluated by pushing resistance (Supplementary Table 1, Fig. 3). In CSSL-PRL4, the basal culms showed high compression resistance, and other physical traits were not clear (Supplementary Table 2). On the other hand, the basal culms of NIL-PRL4 did not show a clear difference in compression resistance compared with "Koshihikari", but the strain at the maximum compression resistance increased (Table 1). These results suggest that high PRL part might be contributed by culm physical strength or the strain of culm until breaking under the compression. Culm physical properties might be determined by the degree of NSC accumulation in the culms. High accumulation of NSC in basal culms by prl5 increased pushing resistance (Kashiwagi et al., 2010). Ishimaru et al. (2008) reported that Irt5 improves Young's modulus by remarkably increasing starch content. In the present study, there were different physical properties of basal culms in the CSSL and NIL harboring PRL4, and these lines showed different features of NSC accumulation (Table 1, Supplementary Table 2). In particular, remarkable starch accumulation would directly increase culm physical strength, not the degree of strain on compression. Based on the results of NIL-PRL 4 , because PRL 4 did not specifically increase starch in the culms at the fully ripe stage, the degree of NSC increase in the NIL could increase the strain while maintaining culm hardness. Therefore, the difference in strain until breaking under compression is one of the indexes used to evaluate lodging resistance between culms 
with the same compression resistance. The relationship between hardness and flexibility of culms, or the degree of NSC accumulation, is a key target for improving lodging resistance.

Physical properties of culms include strength, stiffness, and ductility. Culm strength has been often assessed in terms of bending moment at breaking (Hirano et al. 2017), which is generally measured by the three-point bending test method. This study estimated bending moment at breaking using the compression test, because the basal internode was very short. Culm stiffness indicates Young's modulus and is calculated by stress and strain in the elastic region. Additionally, culm ductility was assessed by the strain until breaking under physical stress conditions in this study. The basal culms' increasing strength or ductility contributed to high pushing resistance, and therefore toughness, by increasing these indicators, which enhanced the strength of the lower part. Culms with high stiffness do not necessarily improve pushing resistance (Kashiwagi et al. 2008). The improvement of pushing resistance should target strength or ductility rather than stiffness in basal culms. At the same culm diameter, NSC accumulation would be the target for improving strength or ductility (Table 1, Supplementary Table 2). In structural carbohydrates, high holocellulose accumulation by BSUC11 enhances culm strength (Kashiwagi et al. 2016). Because carbohydrate accumulation contributes to the determination of physical properties in culms, genetic factors for carbohydrate accumulation are an important tool for breeding to improve lodging resistance. Ookawa and Ishihara (1992) proposed three targets for lodging resistance: bending moment at breaking, section modulus, and bending stress. Section modulus is determined by culm morphology, including diameter, culm wall thickness, and thickness of cortical fiber tissue, and bending stress is calculated from bending moment at breaking and section modulus. For these indicators, SCM2 and SCM3 increase section modulus and improve culm strength (Yano et al. 2015). Additionally, the smos 1 mutation increases culm diameter and culm wall thickness and improves lodging resistance (Hirano et al. 2014). For improvement of lodging resistance, therefore, the determinants of carbohydrate accumulation and morphology in culms and the relationships between these determinants and physical properties require further study.

In conclusion, this study identified a lodging resistance QTL, PRL4, for improving the strength of the lower part of the rice plant. Among physical properties, PRL 4 increased the strain of basal culm until breaking under the compression caused by high NSC accumulation. The genetic control of NSC accumulation in culms may improve lodging resistance by enhancing culm toughness with strength and ductility. Further study including narrowing PRL4 region will be required to reveal the determination mechanisms of culm physical properties and pushing resistance with NSC accumulation in rice.

\section{Abbreviations}

CSSL Chromosomal segment substitution line

NIL Near isogenic line

NSC Non-structural carbohydrate 
PRL Pushing resistance of the lower part

PRL/TN Pushing resistance of the lower part per tiller

QTL quantitative trait locus

\section{Declarations}

Ethics approval and consent to participate Not Applicable.

Consent for publication Not Applicable.

Availability of data and material The datasets during and/or analysed during the current study available from the corresponding author on reasonable request.

Conflict of interest The author declares that there are no conflicts of interest.

Funding This work was supported by JSPS KAKENHI Grant Number JP20K05993.

Authors' contributions The concept, experimental design, analysis, writing, and revision of the manuscript were conducted by TK.

Acknowledgements Not Applicable.

\section{References}

1. Bergmeyer HU, Bernt E (1974) Methods for determination of metabolites: carbohydrate metabolism: sucrose. In: Bergmeyer HU (ed) Methods of Enzymatic Analysis, 3. Academic Press, New York, pp 1176-1179

2. Fan C, Li Y, Hu Z, Hu H, Wang G, Li A, Wang Y, Tu Y, Xia T, Peng L, Feng S (2018) Ectopic expression of a novel OsExtensin-like gene consistently enhances plant lodging resistance by regulating cell elongation and cell wall thickening in rice. Plant Biotechnol J 16:254-263

3. Guo Z, Liu X, Zhang B, Yuan X, Xing Y, Liu H, Luo L, Chen G, Xiong L (2021) Genetic analyses of lodging resistance and yield provide insights into post-Green Revolution breeding in rice. Plant Biotechnol J 19:814-829

4. Hirano K, Kotake T, Kamihara K, Tsuna K, Aohara T, Kaneko Y, Takatsuji H, Tsumuraya Y, Kawasaki S (2010) Rice BRITTLE CULM 3 (BC3) encodes a classical dynamin OsDRP2B essential for proper secondary cell wall synthesis. Planta 232:95-108

5. Hirano K, Okuno A, Hobo T, Ordonio R, Shinozaki Y, Asano K, Kitano H, Matsuoka M (2014) Utilization of stiff culm trait of rice smos1 mutant for increased lodging resistance. PLOS ONE 9:e96009

6. Hirano K, Ordonio RL, Matsuoka M (2017) Engineering the lodging resistance mechanism of postGreen Revolution rice to meet future demands. Proc Jpn Acad Ser B 93:220-233 
7. International Rice Genome Sequencing Project (2005) The map-based sequence of the rice genome. Nature 436:793-800

8. Ishimaru K, Yano M, Aoki N, Ono K, Hirose T, Lin SY, Monna L, Sasaki T, Ohsugi R (2001) Toward the mapping of physiological and agronomic characters on a rice function map: QTL analysis and comparison between QTLs and expressed sequence tags. Theor Appl Genet 102:793-800

9. Ishimaru K, Togawa E, Ookawa T, Kashiwagi T, Madoka Y, Hirotsu N (2008) New target for rice lodging resistance and its effect in a typhoon. Planta 227:601-609

10. Kashiwagi T, Ishimaru K (2004) Identification and functional analysis of a locus for improvement of lodging resistance in rice. Plant Physiol 134:676-683

11. Kashiwagi T, Madoka Y, Hirotsu N, Ishimaru K (2006) Locus prl5 improves lodging resistance of rice by delaying senescence and increaseing carbohydrate reaccumulation. Plant Physiol Biochem 44:152-157

12. Kashiwagi T, Togawa E, Hirotsu N, Ishimaru K (2008) Improvement of lodging resistance with QTLs for stem diameter in rice (Oryza sativa L.). Theor Appl Genet 117:749-757

13. Kashiwagi T, Hirotsu N, Ujiie K, Ishimaru K (2010) Lodging resistance locus pr/5 improves physical strength of the lower plant part under different conditions of fertilization in rice (Oryza sativa L.). Field Crops Res 115:107-115

14. Kashiwagi T (2014) Identification of quantitative trait loci for resistance to bending-type lodging in rice (Oryza sativa L.). Euphytica 198:353-367

15. Kashiwagi T, Munakata J, Ishimaru K (2016) Functional analysis of the lodging resistance QTL BSUC11 on morphological and chemical characteristics in upper culms of rice. Euphytica 210:233243

16. Kono M (1995) Physiological aspects of lodging. In: Matsuo T, Kumazawa K, Ishii R, Ishihara K, Hirata H (eds) Science of the Rice Plant, vol 2. Physiology. Food and Agriculture Policy Research Center, Tokyo, pp 971-982

17. Li X, Yang Y, Yao J, Chen G, Li X, Zhan Q, Wu C (2009) FLEXIBLE CULM1 encording a cinnamylalcohol dehydrogenase controls culm mechanical strength in rice. Plant Mol Biol 69:685-697

18. Madoka Y, Kashiwagi T, Hirotsu N, Ishimaru K (2008) Indian rice "Kasalath" contains genes that improve traits of Japanese premium rice "Koshihikari". Theor Appl Genet 116:603-612

19. Merugumala GR, Satyanarayana PV, Narne C, Ravikumar BNVSR, Ramana Rao PV, Pavani L, Deepika V (2019) Molecular breeding of "Swarna", a mega rice variety for lodging resistance. Mol Breed 39:55

20. Monna L, Kitazawa N, Yoshino R, Suzuki J, Masuda H, Maehara Y, Tanji M, Sato M, Nasu S, Minobe Y (2002) Positional cloning of rice semidwarfing gene, sd-1: rice "green revolution gene" encodes a mutant enzyme involved in gibberellin synthesis. DNA Res 9:11-17

21. Nomura T, Arakawa N, Yamamoto T, Ueda T, Adachi S, Yonemaru J-i et al (2019) Next generation long-culm rice with superior lodging resistance and high grain yield, Monster Rice 1. PLoS ONE 14:e0221424 
22. Ookawa T, Ishihara K (1992) Varietal difference of physical characteristics of the culm related to lodging resistance in paddy rice. Jpn J Crop Sci 61:419-425

23. Ookawa T, Hobo T, Yano M, Murata K, Ando T, Miura H, Asano K, Ochiai Y, Ikeda M, Nishitani R, Ebitani T, Ozaki H, Angeles ER, Hirasawa T, Matsuoka M (2010a) New approach for rice improvement using a pleiotropic QTL gene for lodging resistance and yield. Nat Commun 1:132

24. Ookawa T, Yasuda K, Kato H, Sakai M, Seto M, Sunaga K, Motobayashi T, Tojo S, Hirasawa T (2010b) Biomass production and lodging resistance in 'Leaf Star', a new long-culm rice forage cultivar. Plant Prod Sci 13:58-66

25. Peng S, Cassman K, Virmani SS, Sheehy J, Khush GS (1999) Yield potential trends of tropical rice since the release of IR8 and the challenge of increasing rice yield potential. Crop Sci 39:1552-1559

26. Sowadan O, Li D, Zhang Y, Zhu S, Hu X, Bhanbhro LB, Edzesi WM, Dang X, Hong D (2018) Mining of favorable alleles for lodging resistance traits in rice (oryza sativa) through association mapping. Planta 248:155-169

27. Suzuki S, Suzuki Y, Yamamoto N, Hattori T, Sakamoto M, Umezawa T (2009) High-throughput determination of thioglycolic acid lignin from rice. Plant Biotechnol 26:337-340

28. Takai T, Nonoue Y, Yamamoto S, Yamanouchi U, Matsubara K, Liang ZW, Lin H, Ono N, Uga Y, Yano M (2007) Development of chromosome segment substitution lines derived from backcross between indica donor rice cultivar 'Nona Bokra' and japonica recipient cultivar 'Koshihikari'. Breed Sci 57:257261

29. Terashima K, Ogata T, Akita S (1994) Eco-physiological characteristics related with lodging tolerance of rice in direct sowing cultivation. II. Root growth characteristics of tolerant cultivars to root lodging. Jpn J Crop Sci 63:34-41

30. Ujiie K, Kashiwagi T, Ishimaru K (2012) Identification and functional analysis of alleles for productivity in two sets of chromosome segment substitution lines of rice. Euphytica 187:325-337

31. Wise LE, Murphy M, D'Addieco AA (1946) Chlorite holocellulose, its fractionation and bearing on summative wood analysis and studies on the hemicelluloses. Paper Trade J 122:35-43

32. Yamauchi K, Yamamoto T, Segami S, Horikawa M, Chaya G, Kitano H, Iwasaki Y, Miura K (2020) gw2 mutation increases grain width and culm thickness in rice (Oryza sativa L.). Breed Sci 70:456-461

33. Yano K, Ookawa T, Aya K, Ochiai Y, Hirasawa T, Ebitani T, Takarada T, Yano M, Yamamoto T, Fukuoka $\mathrm{S}, \mathrm{Wu}$ J, Ando T, Ordonio RL, Hirano K, Matsuoka M (2015) Isolation of a novel lodging resistance QTL gene involved in Strigolactone signaling and its pyramiding with a QTL gene involved in another mechanism. Mol Plant 8:303-314

\section{Figures}


a

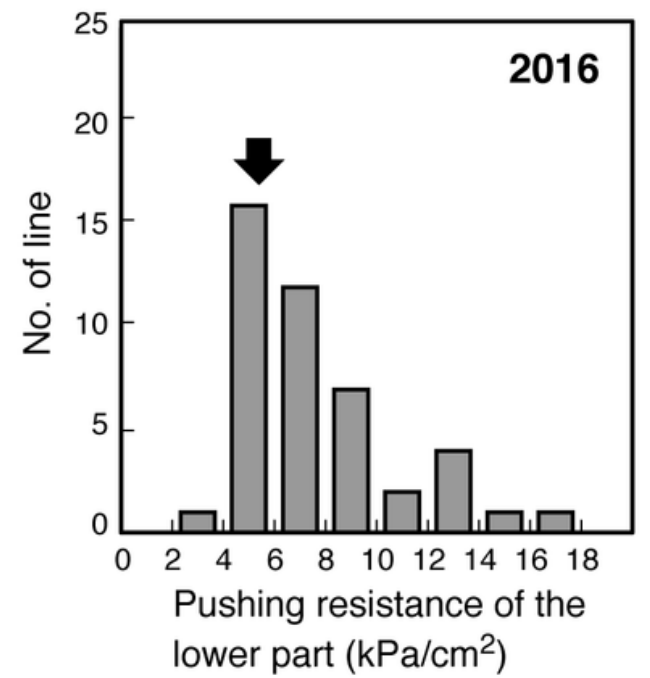

d

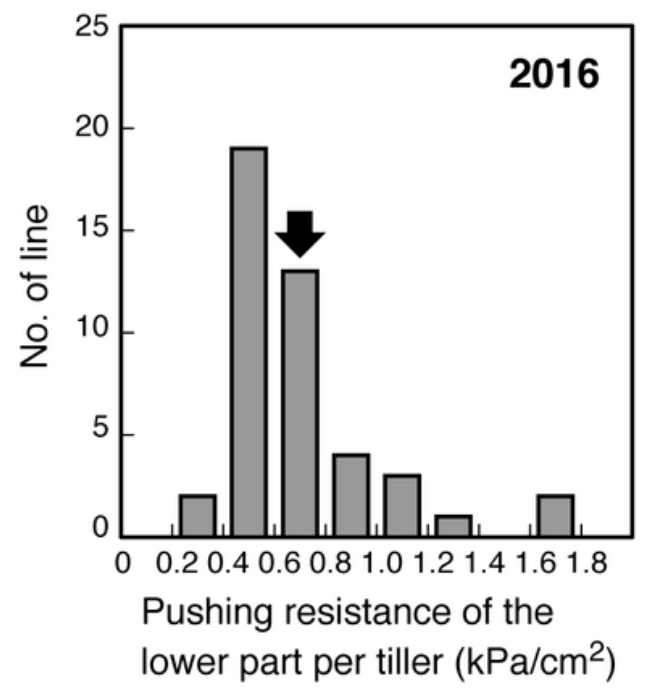

b

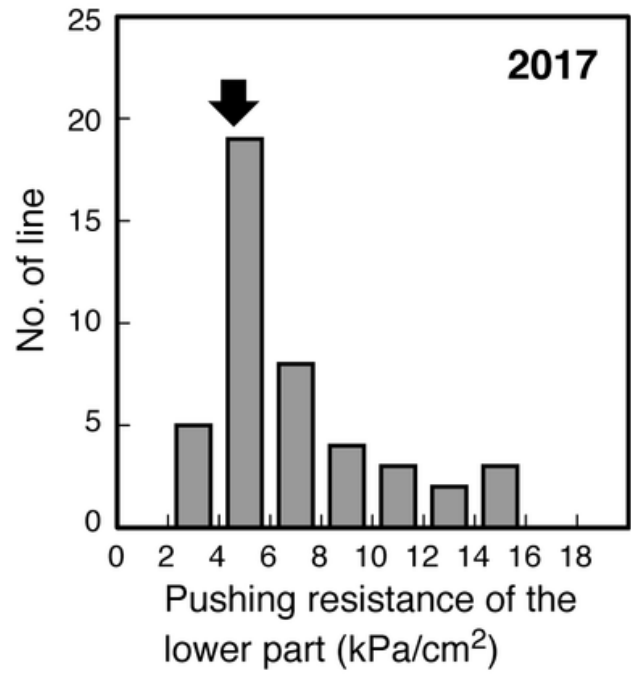

e

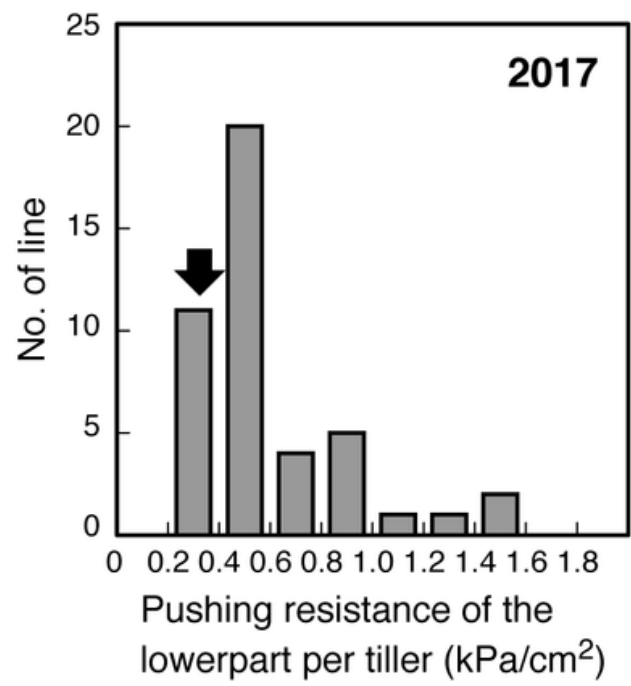

C

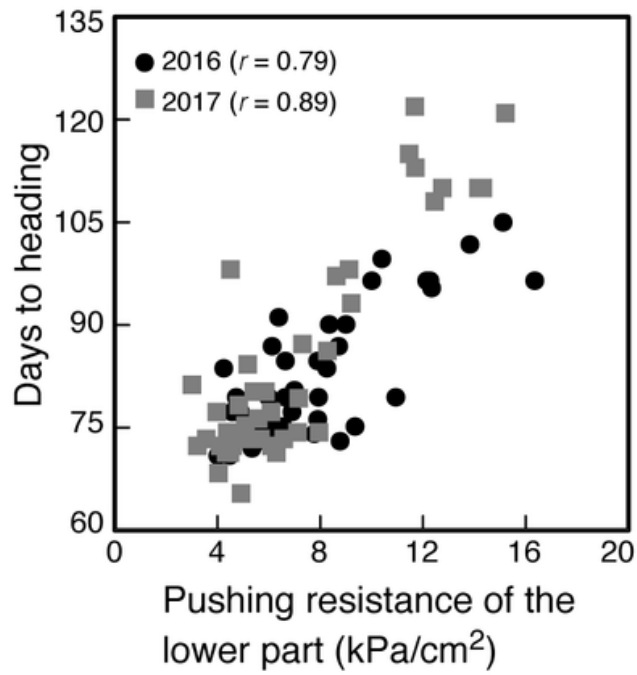

lower part $\left(\mathrm{kPa} / \mathrm{cm}^{2}\right)$

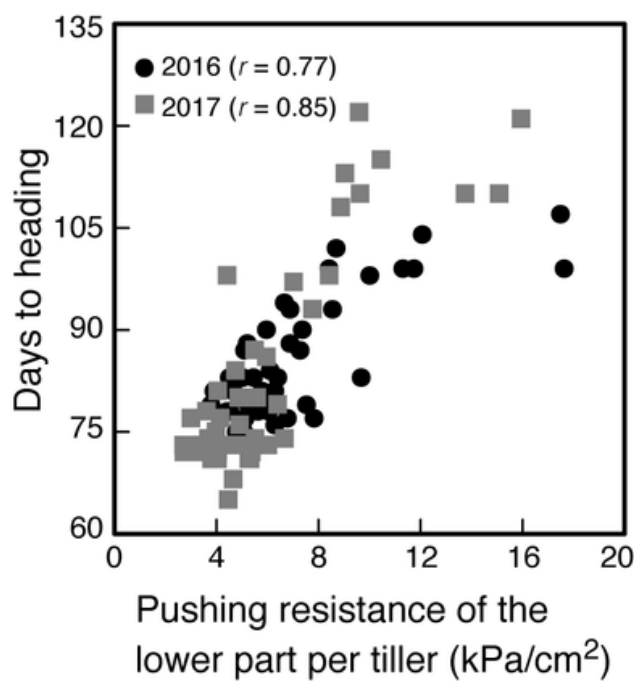

Figure 1

Frequency distributions of pushing resistances in 44 CSSLs at 7 weeks after heading and correlations between pushing resistance and days to heading. (a) and (b) show the histogram of PRL; (d) and (e) show the histogram of PRL/TN. (c) and (f) show the correlation. Black arrows indicate the mean values for "Koshihikari" 

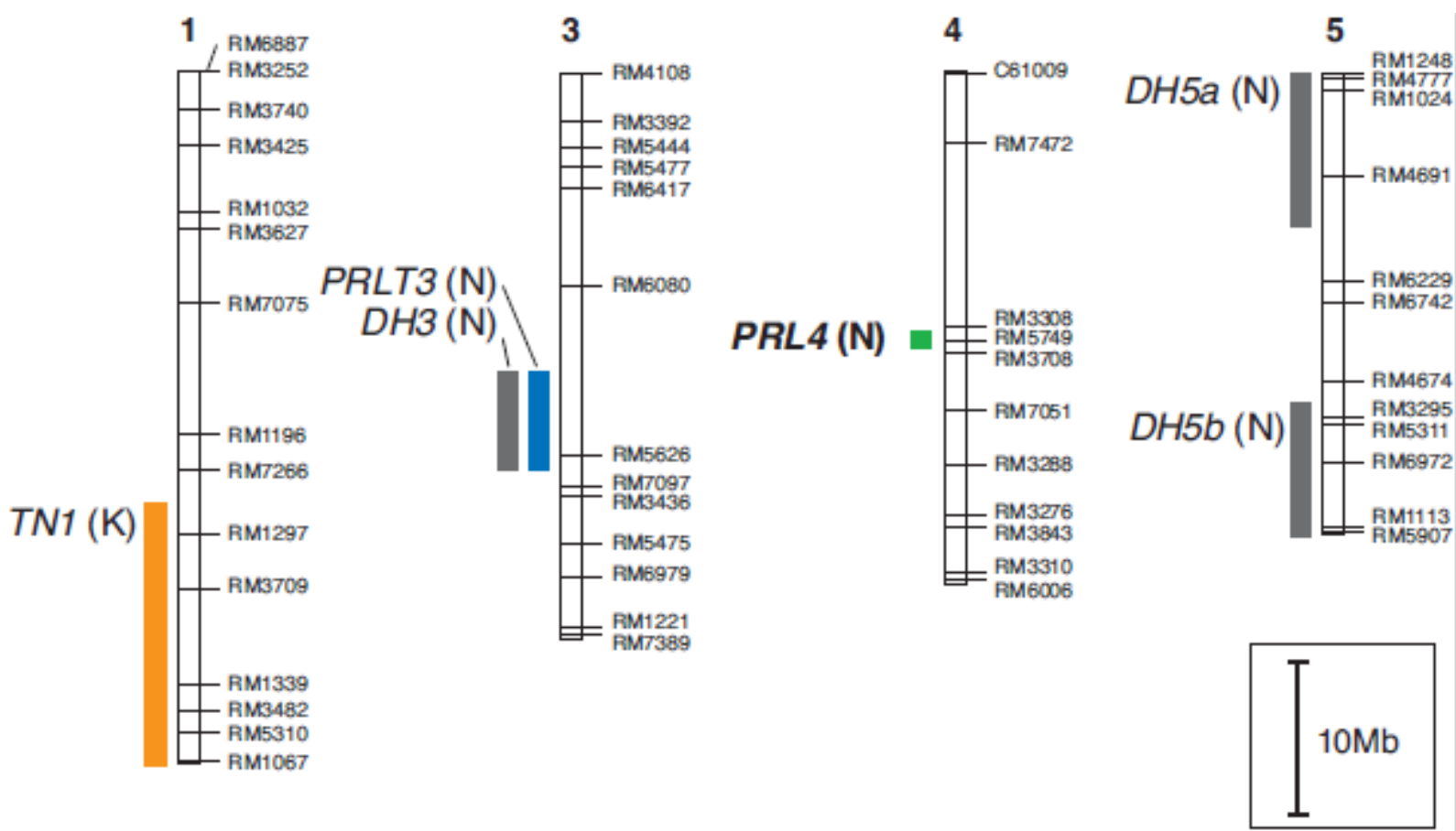

6

7
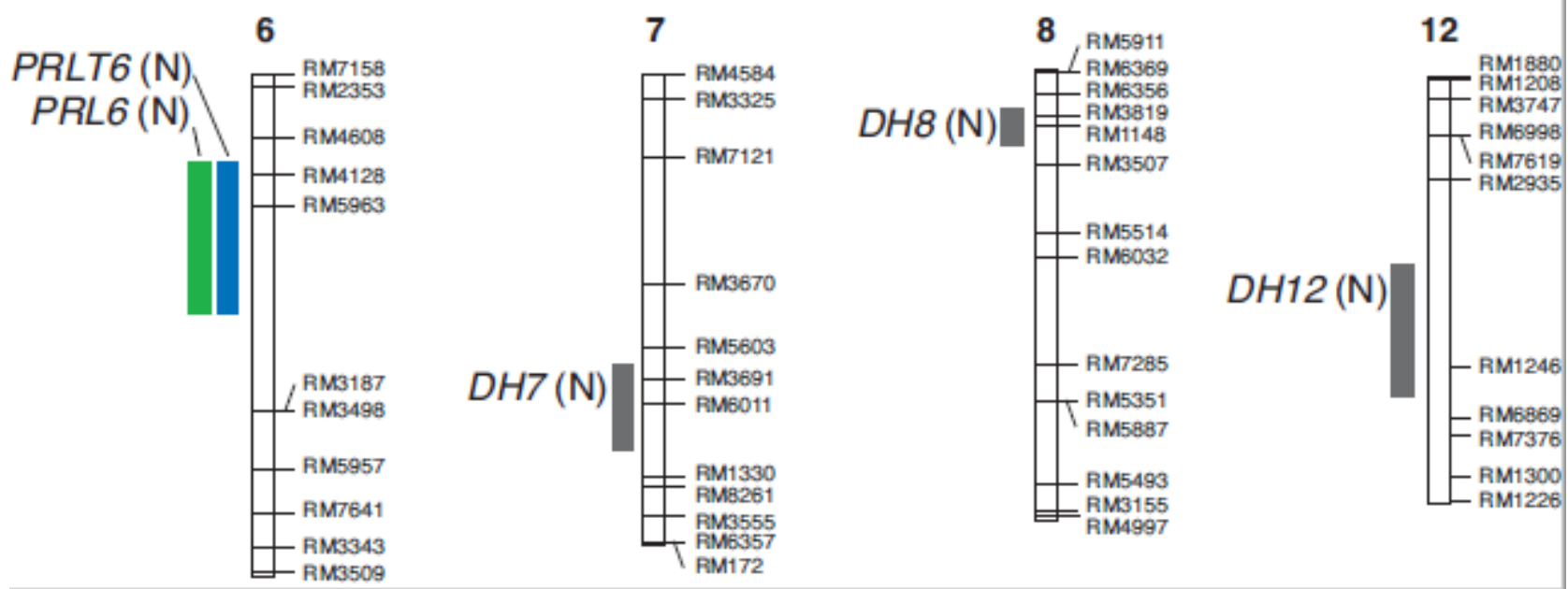

\section{Figure 2}

Map locations of QTLs for pushing resistance, tiller number, and days to heading. Simple sequence repeat (SSR) markers were defined by McCouch et al. (2002). Mapped QTLs indicate the locus detected at the same position in two years. K and $\mathrm{N}$ in parentheses indicate that "Koshihikari" and "Nona Bokra" alleles have a positive effect, respectively 
a

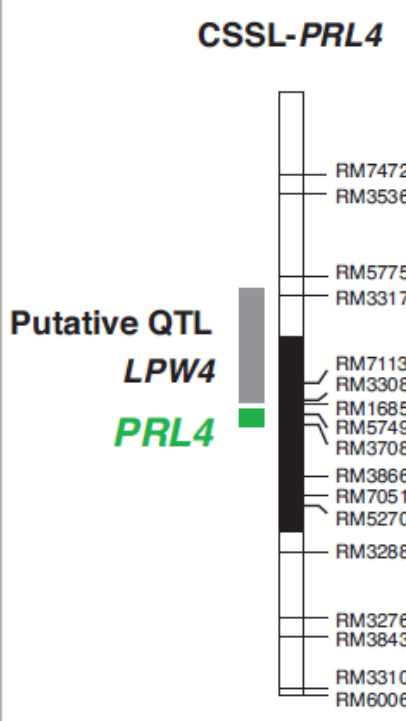

(10.3Mb)
NIL-PRL4

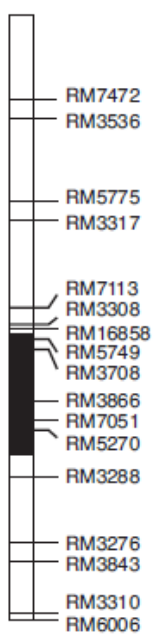

(6.6Mb) b

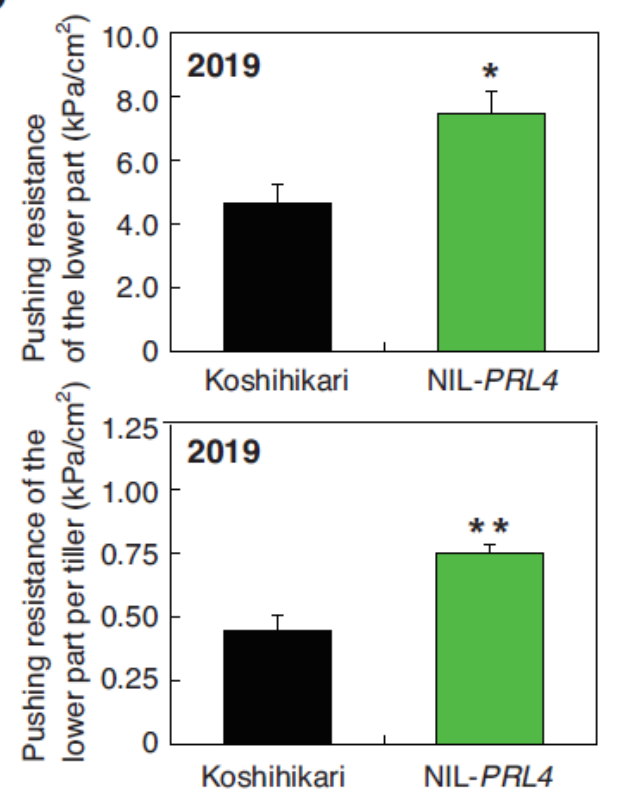

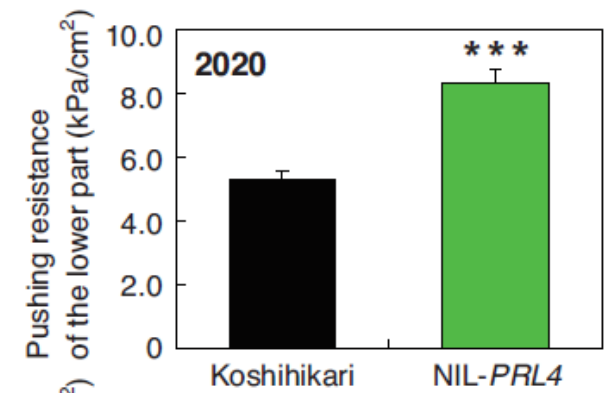

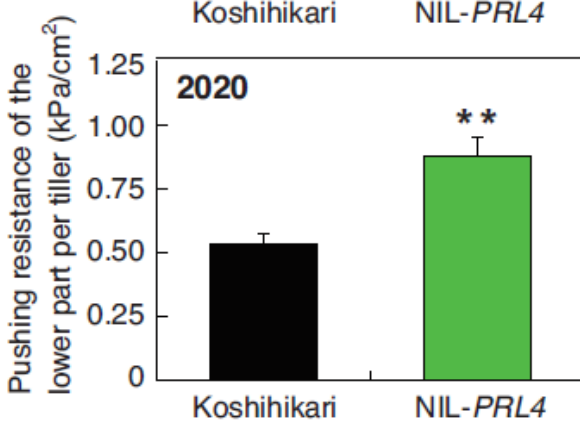

\section{Figure 3}

Graphical genotypes on chromosome 4 in CSSL-PRL4 and NIL-PRL4 (a) and pushing resistances of "Koshihikari" and NIL-PRL4 (b). LPW4 locus is the QTL for low panicle weight reported by Ujiie et al. (2012). Vertical bars on pushing resistances indicate standard errors. ***, **, and * indicate significant differences at $0.001,0.01$, and 0.05 probability levels, respectively 